\title{
Pacific
}

Journal of

Mathematics

\section{PAINLEVÉ ANALYSIS OF GENERALIZED ZAKHAROV EQUATIONS}

\author{
Hassan A. Zedan and SAlma M. Al-Tuwairqi
}




\title{
PAINLEVÉ ANALYSIS OF GENERALIZED ZAKHAROV EQUATIONS
}

\author{
Hassan A. Zedan And Salma M. Al-Tuwairqi
}

We consider the invariance and integrability properties of the generalized Zakharov equations and obtain an exact invariant solution. We derive the Painlevé property of these equations and obtain some exact solutions using the truncated Painlevé expansion.

\section{Introduction}

We consider the generalized Zakharov equations (GZEs) for the complex envelope $E(x, t)$ of the high-frequency wave and the real low-frequency field $\eta(x, t)$ in the form

$$
\begin{aligned}
& i E_{t}+E_{x x}-2 \beta|E|^{2} E+2 E \eta=0, \\
& \eta_{t t}-\eta_{x x}=-\left(|E|^{2}\right)_{x x},
\end{aligned}
$$

where the cubic term in Equation (1) describes nonlinear self-interaction in the high-frequency subsystem; such a term corresponds to a self-focusing effect in plasma physics. The coefficient $\beta$ is a real constant that can be positive or negative. The sound velocity and the coupling constant in Equation (2) have been normalized to unity for simplicity. The GZEs are a universal model of interaction between high- and low-frequency waves in one dimension. The collisions between solitary waves of GZEs have been simulated in detail in [Hadouaj et al. 1991]. When $\beta=0$, this system is reduced to the classical Zakharov equations for plasma physics.

Several aspects of the GZEs have been studied. Malomed, Anderson, Lisak, Quiroga-Teixeiro and Stenflo [1997] analyzed them using a variational approach. Wang and Li [2005] introduced periodic wave solutions using the extended $F$ expansion method. By considering the modified Adomian decomposition method, Wang, Dai, Wu, Lei and Zhang [2007] calculated exact and numerical solutions. Zhang [2007] constructed exact traveling wave solutions by a direct algebraic method. Li, Li and Lin [2008] used the exp-function method to obtain exact solutions. Javidi and Golbabi [2008] obtained exact and numerical solutions by the

MSC2000: primary 35Q51; secondary 35K05.

Keywords: generalized Zakharov equations, Lie symmetries, Painlevé expansion. 
variational iteration method. Abbasbandy, Babolian and Ashtiani [2009] applied homotopy analysis method to obtain a solution.

The objective of this work is to investigate the symmetry and conduct Painlevé analysis of GZEs. It will be organized as follows. In Section 2, we obtain the Lie point symmetry group of GZEs and its similarity reductions. Moreover, we also construct explicit analytic invariant solutions. In Section 3, we inspect the singularity structure of GZEs by means of the Weiss-Tabor-Carnevale procedure. In Section 4, we obtain some exact solutions using the truncated Painlevé expansion.

\section{Symmetry analysis}

Symmetry is one of the most important concepts in the area of partial differential equations [Bluman and Kumei 1989]. To find the Lie symmetries of the GZEs, we express the complex envelope as $E(x, t)=u(x, t)+i v(x, t)$, with real highfrequency waves $u(x, t)$ and $v(x, t)$. Substituting it into Equations (1) and (2) and separating the imaginary and real parts we obtain the equations

$$
\begin{array}{r}
u_{x x}-v_{t}-2 \beta\left(u^{2}+v^{2}\right) u+2 \eta u=0, \\
u_{t}+v_{x x}-2 \beta\left(u^{2}+v^{2}\right) v+2 \eta v=0, \\
\eta_{t t}-\eta_{x x}=-\left(u^{2}+v^{2}\right)_{x x} .
\end{array}
$$

Consider a one-parameter Lie group of infinitesimal transformations of the form

$$
\begin{aligned}
u \rightarrow U & =u+\varepsilon \phi_{1}(x, t, u, v, \eta), \\
v \rightarrow V & =v+\varepsilon \phi_{2}(x, t, u, v, \eta), \\
\eta \rightarrow \Lambda & =\eta+\varepsilon \phi_{3}(x, t, u, v, \eta), \\
x \rightarrow X & =x+\varepsilon \xi_{1}(x, t, u, v, \eta), \\
t \rightarrow T & =t+\varepsilon \xi_{2}(x, t, u, v, \eta) \quad \text { for } \varepsilon \ll 1,
\end{aligned}
$$

with infinitesimal generator

$$
\widetilde{X}=\xi_{1} \frac{\partial}{\partial x}+\xi_{2} \frac{\partial}{\partial t}+\phi_{1} \frac{\partial}{\partial u}+\phi_{2} \frac{\partial}{\partial v}+\phi_{3} \frac{\partial}{\partial \eta} .
$$

Require that (3) are invariant under (4) by direct substitution [Bluman and Kumei 1989]. Eliminate $u_{t}, v_{t}, \eta_{t t}$ using (3), and set to zero all coefficients of the independent terms of the polynomials of $u, v$ and $\eta$ and their partial derivatives. We then obtain the determining equations for the infinitesimals. By solving these equations 
we have

$$
\begin{aligned}
& \phi_{1}=-\left(k_{3}+k_{4} t+k_{5} t^{2}\right) v, \\
& \phi_{2}=\left(k_{3}+k_{4} t+k_{5} t^{2}\right) u, \\
& \phi_{3}=\frac{1}{2} k_{4}+k_{5} t, \\
& \xi_{1}=k_{1}, \quad \xi_{2}=k_{2},
\end{aligned}
$$

where $k_{1}, k_{2}, k_{3}, k_{4}$ and $k_{5}$ are arbitrary parameters. The infinitesimal generator of the Lie algebra associated with each parameter $k_{i}$ is obtained from the generator (5):

$$
\begin{array}{ll}
L_{1}=\frac{\partial}{\partial x}, \quad L_{2}=\frac{\partial}{\partial t}, & L_{4}=t u \frac{\partial}{\partial v}-t v \frac{\partial}{\partial u}+\frac{1}{2} \frac{\partial}{\partial \eta}, \\
L_{3}=u \frac{\partial}{\partial v}-v \frac{\partial}{\partial u}, & L_{5}=t^{2} u \frac{\partial}{\partial v}-t^{2} v \frac{\partial}{\partial u}+t \frac{\partial}{\partial \eta} .
\end{array}
$$

The similarity solution can be obtained from the invariant surface equation

$$
\frac{d t}{k_{2}}=\frac{d x}{k_{1}}=\frac{d u}{-\left(k_{3}+k_{4} t+k_{5} t^{2}\right) v}=\frac{d v}{\left(k_{3}+k_{4} t+k_{5} t^{2}\right) u}=\frac{d \eta}{k_{4} / 2+k_{5}} .
$$

For the most general generator $X$, we obtain the similarity solution

$$
\begin{aligned}
& u(x, t)=c \cos \left(\frac{k_{5}}{3 k_{2}} t^{3}+\frac{k_{4}}{2 k_{2}} t^{2}+\frac{k_{3}}{k_{2}} t+F_{1}(z)\right), \\
& v(x, t)=c \sin \left(\frac{k_{5}}{3 k_{2}} t^{3}+\frac{k_{4}}{2 k_{2}} t^{2}+\frac{k_{3}}{k_{2}} t+F_{1}(z)\right), \\
& \eta(x, t)=\frac{k_{5}}{2 k_{2}} t^{2}+\frac{k_{4}}{2 k_{2}} t+F_{2}(z),
\end{aligned}
$$

where $F_{1}$ and $F_{2}$ are similarity functions of the similarity variable $z=k_{1} t-k_{2} x$, satisfying the similarity equations

$$
\begin{aligned}
& 0=-k_{2}^{3}\left(F_{1}^{\prime \prime}-\left(F_{1}^{\prime}\right)^{2}\right)+k_{1} k_{2} F_{1}^{\prime}-2 k_{2} F_{2}+k_{3}+2 c^{2} \beta k_{2}, \\
& 0=k_{2}^{3}\left(F_{1}^{\prime \prime}+\left(F_{1}^{\prime}\right)^{2}\right)+k_{1} k_{2} F_{1}^{\prime}-2 k_{2} F_{2}+k_{3}+2 c^{2} \beta k_{2}, \\
& 0=F_{2}^{\prime \prime}+k_{5} /\left(k_{2}\left(k_{1}^{2}-k_{2}^{2}\right)\right),
\end{aligned}
$$

where prime denotes differentiation with respect to $z$. Solving equations (6), we obtain the solution

$$
\begin{aligned}
& F_{1}=\frac{1}{2 k_{2}^{3}}\left(-k_{1} k_{2}+\sqrt{\left(k_{1} k_{2}\right)^{2}-4 k_{2}^{3}\left(k_{3}-2 k_{2}\left(a_{2}-c^{2} \beta\right)\right)}\right) z+a_{0}, \\
& F_{2}=a_{1},
\end{aligned}
$$

where $a_{0}$ and $a_{1}$ are arbitrary constants. Hence the exact invariant solution to 
GZEs (1) and (2) can be written as

$$
\begin{aligned}
E(x, t)=c \exp ( & \frac{i}{2 k_{2}^{3}}\left(k_{2}^{2}\left(\frac{2}{3} k_{5} t^{3}+k_{4} t^{2}+2 k_{3} t\right)\right. \\
& \left.\left.\quad+\left(k_{1} t-k_{2} x\right) \sqrt{\left(k_{1} k_{2}\right)^{2}-4 k_{2}^{3}\left(k_{3}-2 k_{2}\left(a_{1}-c^{2} \beta\right)\right)}-k_{1} k_{2}+2 k_{2}^{3} a_{0}\right)\right), \\
\eta(x, t)= & \frac{k_{5}}{2 k_{2}} t^{2}+\frac{k_{4}}{2 k_{2}} t+a_{1} .
\end{aligned}
$$

\section{Painlevé analysis}

The Painlevé test is one of the impressive ways to test whether partial differential equations are integrable or nonintegrable. Myrzakulov [1999] has confirmed the Painlevé nature of the $(2+1)$-dimensional Zakharov equation. In this paper we will show that GZEs possess the Painlevé property. Various approaches can be applied to investigate the Painlevé integrability. Here we will use WTC method [Weiss et al. 1983]. Consider Laurent expansion of the solutions of (3) in a local neighborhood of a movable singular manifold $\phi(x, t)=0$ :

$$
u(x, t)=\sum_{j=0}^{\infty} u_{j} \phi^{j+\alpha_{1}}, \quad v(x, t)=\sum_{j=0}^{\infty} v_{j} \phi^{j+\alpha_{2}}, \quad \eta(x, t)=\sum_{j=0}^{\infty} \eta_{j} \phi^{j+\alpha_{3}},
$$

where $u_{j}(x, t), v_{j}(x, t)$ and $\eta_{j}(x, t)$ are analytic functions and the three $\alpha_{i}$ are integers to be determined. It is sufficient to substitute

$$
u(x, t)=u_{0} \phi^{\alpha_{1}}, \quad v(x, t)=v_{0} \phi^{\alpha_{2}}, \quad \eta(x, t)=\eta_{0} \phi^{\alpha_{3}},
$$

into equations (3) to find the dominant behavior and leading exponents $\alpha_{i}$. Balancing the dominant terms, we get $\alpha_{1}=\alpha_{2}=-1$ and $\alpha_{3}=-2$, and the equations

$$
u_{0}^{2}+v_{0}^{2}=\phi_{x}^{2}-\frac{\phi_{x}^{4}}{\beta^{2} \phi_{t}^{2}+\beta(1-\beta) \phi_{x}^{2}} \quad \text { and } \quad \eta_{0}=-\frac{\phi_{x}^{4}}{\beta^{2} \phi_{t}^{2}+(1-\beta) \phi_{x}^{2}} .
$$

The first shows that either $u_{0}$ or $v_{0}$ is arbitrary. Substituting the series expansion

$$
u=u_{0} \phi^{-1}+u_{r} \phi^{r-1}, \quad v=v_{0} \phi^{-1}+v_{r} \phi^{r-1}, \quad \eta=\eta_{0} \phi^{-2}+\eta_{r} \phi^{r-2},
$$

into equations (3) and collecting the coefficients of $u_{r}, v_{r}$ and $\eta_{r}$ for leading terms, the resonance are found to have the values $r=-1,0,2,3,3,4$. As usual, the resonance $r=-1$ represents the arbitrariness of the singularity manifold $\phi(x, t)=0$, and the remaining resonance values indicate the arbitrariness of five functions (one of $\left\{u_{0}, v_{0}, \eta_{0}\right\}$, one of $\left\{u_{2}, v_{2}, \eta_{2}\right\}$, two of $\left\{u_{3}, v_{3}, \eta_{3}\right\}$ and one of $\left.\left\{u_{4}, v_{4}, \eta_{4}\right\}\right)$. 


\section{Some solutions to the generalized Zakharov equation}

When an equation has the Painlevé property, one can find some kinds of exact solutions by truncating the Painleve expansion of the solution about the movable singularity manifold at the constant level term, leading to the so-called Backlund transformation of the equation. For GZEs, the transformation has the form

$$
u=u_{0} \phi^{-1}+u_{1}, \quad v=v_{0} \phi^{-1}+v_{1}, \quad \eta=\eta_{0} \phi^{-2}+\eta_{1} \phi^{-1}+\eta_{2} .
$$

Our approach to finding an exact solution for $u, v$ and $\eta$ is similar to that of [Choudhury 2006]. Substituting equations (7) into equations (3) and equating the coefficients of $\phi^{-i}$ for $i=0,1,2,3,4$ to zero, we obtain this system of PDEs:

(8) $0=-2 \beta u_{1}^{2} v_{1}-2 \beta v_{1}^{3}+2 v_{1} \eta_{2}+u_{1, t}+v_{1, x x}$,

(9) $0=-2 \beta u_{1}^{2} v_{0}-4 \beta u_{0} u_{1} v_{1}-6 \beta v_{0} v_{1}^{2}+2 v_{1} \eta_{1}+2 v_{0} \eta_{2}+u_{0, t}+v_{0, x x}$,

(10) $0=-4 \beta u_{0} u_{1} v_{0}-2 \beta u_{0}^{2} v_{1}-6 \beta v_{0}^{2} v_{1}+2 v_{1} \eta_{0}+2 v_{0} \eta_{1}-u_{0} \phi_{t}-2 v_{0, x} \phi_{x}-v_{0} \phi_{x x}$,

(11) $0=-2 \beta u_{0}^{2} v_{0}-2 \beta v_{0}^{3}+2 v_{0} \eta_{0}+2 v_{0} \phi_{x}^{2}$,

(12) $0=-2 \beta u_{1}^{3}-2 \beta u_{1} v_{1}^{2}+2 u_{1} \eta_{2}-v_{1, t}+u_{1, x x}$,

(13) $0=-6 \beta u_{0} u_{1}^{2}-4 \beta u_{1} v_{0} v_{1}-2 \beta u_{0} v_{1}^{2}+2 u_{1} \eta_{1}+2 u_{0} \eta_{2}-v_{0, t}+u_{0, x x}$,

(14) $0=-6 \beta u_{0}^{2} u_{1}-2 \beta u_{1} v_{0}^{2}-4 \beta u_{0} v_{0} v_{1}+2 u_{1} \eta_{0}+2 u_{0} \eta_{1}+v_{0} \phi_{t}-2 u_{0, x} \phi_{x}-u_{0} \phi_{x x}$,

(15) $0=-2 \beta u_{0}^{3}-2 \beta u_{0} v_{0}^{2}+2 u_{0} \eta_{0}+2 u_{0} \phi_{x}^{2}$,

(16) $0=\eta_{2, t t}+2 u_{1, x}^{2}+2 v_{1, x}^{2}+2 u_{1} u_{1, x x}+2 v_{1} v_{1, x x}-\eta_{2, x x}$,

(17) $0=\eta_{1, t t}+4 u_{0, x} u_{1, x}+4 v_{0, x} v_{1, x}+2 u_{1} u_{0, x x}+2 u_{0} u_{1, x x}$

$$
+2 v_{1} v_{0, x x}+2 v_{0} v_{1, x x}-\eta_{1, x x}
$$

(18) $0=-2 \eta_{1, t} \phi_{t}-\eta_{1} \phi_{t t}+\eta_{0, t t}-4 u_{1} \phi_{x} u_{0, x}+2 u_{0, x}^{2}-4 u_{0} \phi_{x} u_{1, x}$

$$
\begin{aligned}
-4 v_{1} \phi_{x} v_{0, x} & +2 v_{0, x}^{2}-4 v_{0} \phi_{x} v_{1, x}+2 \phi_{x} \eta_{1, x}-2 u_{0} u_{1} \phi_{x x} \\
& -2 v_{0} v_{1} \phi_{x x}+\eta_{1} \phi_{x x}+2 u_{0} u_{0, x x}+2 v_{0} v_{0, x x}-\eta_{0, x x},
\end{aligned}
$$

(19) $0=2 \eta_{1} \phi_{t}^{2}-4 \phi_{t} \eta_{0, t}-2 \eta_{0} \phi_{t t}+4 u_{0} u_{1} \phi_{x}^{2}+4 v_{0} v_{1} \phi_{x}^{2}-2 \eta_{1} \phi_{x}^{2}$

$$
-8 u_{0} \phi_{x} u_{0, x}-8 v_{0} \phi_{x} v_{0, x}+4 \phi_{x} \eta_{0, x}-2 u_{0}^{2} \phi_{x x}-2 v_{0}^{2} \phi_{x x}+2 \eta_{0} \phi_{x x},
$$

(20) $0=6 \eta_{0} \phi_{t}^{2}+6 u_{0}^{2} \phi_{x}^{2}+6 v_{0}^{2} \phi_{x}^{2}-6 \eta_{0} \phi_{x}^{2}$.

Substituting a trial solution $\phi(x, t)=1+\exp (i Q(x, t))$ into system (8)-(20), we consider two cases:

Case 1. Let $v_{0}(x, t)=\exp (i Q(x, t))$ be the arbitrary function; then equation (11), (15) and (10) become

$$
\begin{aligned}
& 0=-2 e^{i Q}\left(e^{2 i Q}\left(\beta+Q_{x}^{2}\right)+\beta u_{0}^{2}-\eta_{0}\right), \\
& 0=-2 u_{0}\left(e^{2 i Q}\left(\beta+Q_{x}^{2}\right)+\beta u_{0}^{2}-\eta_{0}\right), \\
& \begin{array}{r}
0=-2 \beta u_{0}^{2} v_{1}+v_{1}\left(-6 \beta e^{2 i Q}+2 \eta_{0}\right)+2 e^{i Q} \eta_{1} \\
\quad-e^{i Q_{0}} u_{0}\left(4 \beta u_{1}+i Q_{t}\right)+3 e^{2 i Q} Q_{x}^{2}-i e^{2 i Q} Q_{x x} .
\end{array}
\end{aligned}
$$


It is convenient to separate (23) into two equations:

$$
\begin{aligned}
& 0=-e^{i Q} u_{0}\left(i Q_{t}\right)-i e^{2 i Q} Q_{x x}, \\
& 0=-2 \beta u_{0}^{2} v_{1}+v_{1}\left(-6 \beta e^{2 i Q}+2 \eta_{0}\right)+2 e^{i Q} \eta_{1}-4 \beta e^{i Q} u_{0} u_{1}+3 e^{2 i Q} Q_{x}^{2} .
\end{aligned}
$$

Solving the first for $u_{0}$ we get $u_{0}(x, t)=-Q_{x x} e^{i Q} / Q_{t}$. Substituting this into (21) we obtain

$$
\eta_{0}(x, t)=\left(\beta Q_{t}^{2}+Q_{t}^{2} Q_{x}^{2}+\beta Q_{x x}^{2}\right) e^{2 i Q} / Q_{t}^{2} .
$$

Letting $Q(x, t)=f_{1}(t) x+f_{2}(t)$ and substituting this into system (8)-(20), we find that (20) has the form

$$
\begin{aligned}
& 0=6 e^{4 i\left(f_{1} x+f_{2}\right)}\left(f_{1}^{4}-\beta\left(x f_{1}^{\prime}+f_{2}^{\prime}\right)^{2}+f_{1}^{2}\left(-1+\beta-x^{2} f_{1}^{\prime 2}-2 x f_{1}^{\prime} f_{2}^{\prime}-f_{2}^{\prime 2}\right)\right), \\
& 0=f_{1}^{4}-\beta\left(x f_{1}^{\prime}+f_{2}^{\prime}\right)^{2}+f_{1}^{2}\left(-1+\beta-x^{2} f_{1}^{\prime 2}-2 x f_{1}^{\prime} f_{2}^{\prime}-f_{2}^{\prime 2}\right) .
\end{aligned}
$$

Equating the coefficients of $x^{2}, x^{1}$ and $x^{0}$ in the second equation to zero, we obtain

$$
\begin{aligned}
& 0=-f_{1}^{2}+\beta f_{1}^{2}+f_{1}^{4}-\beta f_{2}^{\prime 2}-f_{1}^{2} f_{2}^{\prime 2}, \\
& 0=-2 \beta f_{1}^{\prime} f_{2}^{\prime}-2 f_{1}^{2} f_{1}^{\prime} f_{2}^{\prime}, \\
& 0=-f_{1}^{\prime 2}\left(\beta+f_{1}^{2}\right) .
\end{aligned}
$$

The first two are satisfied if $f_{1}(t)=c_{1}$. Substituting this into the last and solving for $f_{2}$, we get

$$
f_{2}(t)=\sqrt{\frac{-c_{1}^{2}\left(c_{1}^{2}+\beta-1\right)}{-\beta-c_{1}^{2}}} t+c_{2} .
$$

Substituting all the above results into equations (10), (13) and (14) and solving for $v_{1}, u_{1}$ and $\eta_{1}$ we find $v_{1}(x, t)=-1 / 2$,

$$
u_{1}(x, t)=\frac{i \sqrt{\left(\beta+c_{1}^{2}\right)\left(\beta+c_{1}^{2}-1\right)}}{2 c_{1}\left(\beta+c_{1}^{2}\right)} \quad \text { and } \quad \eta_{1}(x, t)=-\left(\beta+c_{1}^{2}\right) \exp (i h(x, t)) \text {, }
$$

where $h(x, t)=c_{1} x+c_{2}+\sqrt{c_{1}^{2}\left(1-\beta-c_{1}^{2}\right)} t / \sqrt{-\beta-c_{1}^{2}}$. Solving (8) or (9) or (12) for $\eta_{2}$, we get

$$
\eta_{2}(x, t)=\frac{\beta\left(1-c_{1}^{2}+c_{1}^{4}\right)-\beta^{2}\left(1-c_{1}^{2}\right)}{4 c_{1}^{2}\left(\beta+c_{1}^{2}\right)},
$$

Substituting all the results into the truncated expansion (7), we obtain the corresponding exact solution of GZEs (3):

$$
\begin{aligned}
& u(x, t)=-\frac{i \sqrt{1-\beta-c_{1}^{2}}}{2 c_{1} \sqrt{-\beta-c_{1}^{2}}}, \quad v(x, t)=-\frac{1}{2}+\frac{\exp (i h(x, t))}{1+\exp (i h(x, t))}, \\
& \eta(x, t)=\frac{\beta\left(1-c_{1}^{2}+c_{1}^{4}\right)-\beta^{2}\left(1-c_{1}^{2}\right)}{4 c_{1}^{2}\left(\beta+c_{1}^{2}\right)} \\
&+\frac{\left(\beta+c_{1}^{2}\right) \exp (i h(x, t))}{1+\exp (i h(x, t))}\left(\frac{\exp (i h(x, t))}{1+\exp (i h(x, t))}-1\right) .
\end{aligned}
$$


Figures 1, 2, 3, 4 and 5 illustrate the solution for certain $\beta, c_{1}$ and $c_{2}$.

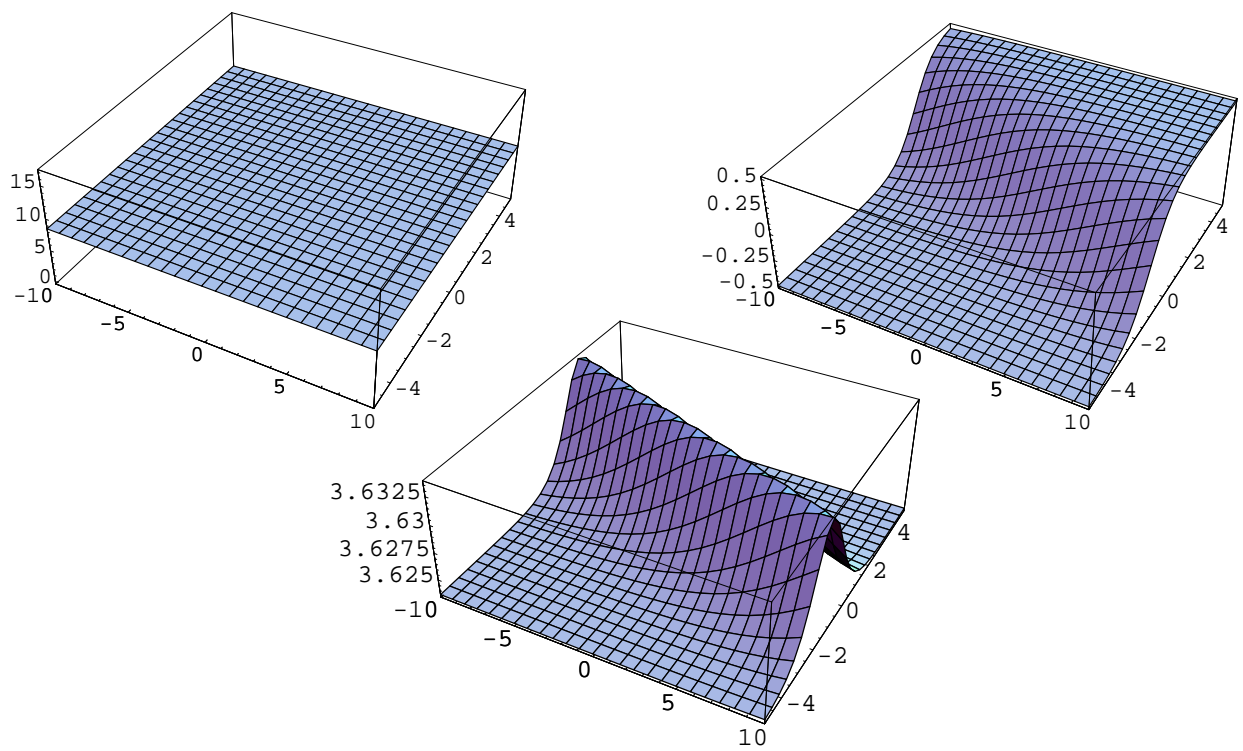

Figure 1. Clockwise from top left: Graphs of $u(x, t), v(x, t)$ and $\eta(x, t)$ for $\beta=0.05, c_{1}=-0.3 i$ and $c_{2}=i$.

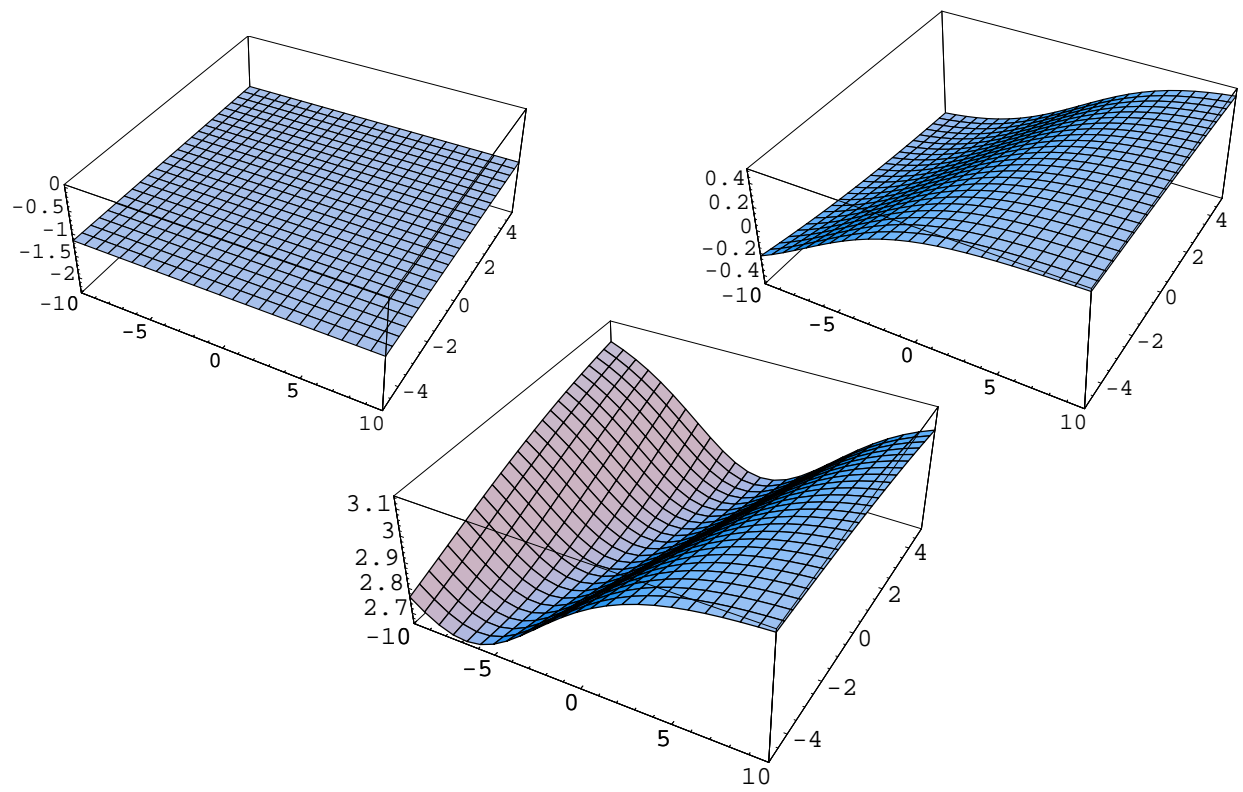

Figure 2. Clockwise from top left: Graphs of $u(x, t), v(x, t)$ and $\eta(x, t)$ for $\beta=2, c_{1}=-0.3 i$ and $c_{2}=-i$. 


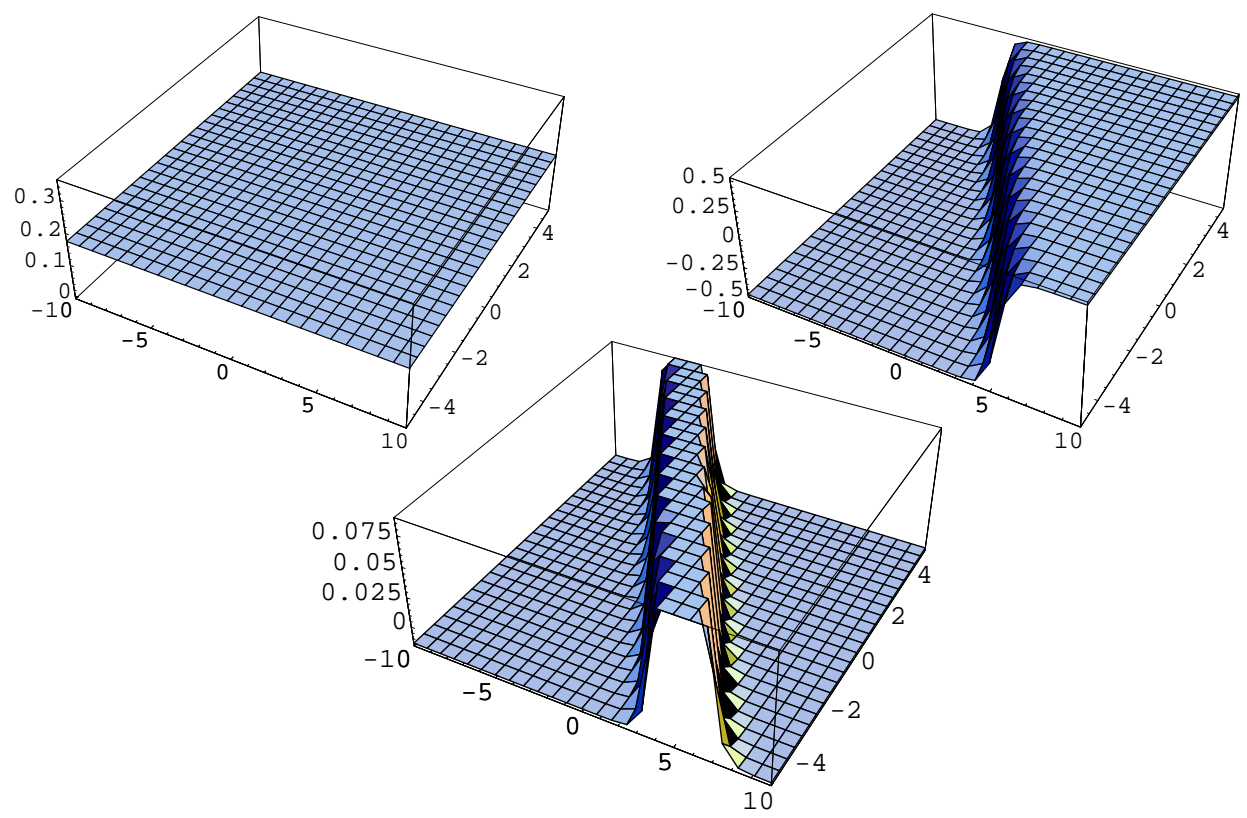

Figure 3. Clockwise from top left: Graphs of $u(x, t), v(x, t)$ and $\eta(x, t)$ for $\beta=-0.05, c_{1}=-3 i$ and $c_{2}=0.4 i$.

Case 2: Let

$$
u_{0}(x, t)=\exp (i Q(x, t))
$$

be the arbitrary function. Then separate (14) into two equations:

$$
\begin{aligned}
& 0=i v_{0} Q_{t}-i e^{i Q} Q_{x x}, \\
& 0=-2 u_{1}\left(3 \beta e^{2 i Q}+\beta v_{0}^{2}-\eta_{0}\right)+e^{i Q}\left(2 \eta_{1}+v_{0}\left(-4 \beta v_{1}+i Q_{t}\right)+3 e^{i Q} Q_{x}^{2}\right) .
\end{aligned}
$$

From the first we obtain $v_{0}(x, t)=Q_{x x} e^{i Q} / Q_{t}$. Solving (11) for $\eta_{0}$, we get

$$
\eta_{0}(x, t)=\left(\beta Q_{t}^{2}+Q_{t}^{2} Q_{x}^{2}+\beta Q_{x x}^{2}\right) e^{2 i} Q / Q_{t}^{2}
$$

Letting $Q(x, t)=f_{1}(t) x+f_{2}(t)$ and substituting this into system (8)-(20), we find that (20) assumes the form

$$
\begin{aligned}
& 0=6 e^{4 i\left(f_{1} x+f_{2}\right)}\left(f_{1}^{4}-\beta\left(x f_{1}^{\prime}+f_{2}^{\prime}\right)^{2}+f_{1}^{2}\left(-1+\beta-x^{2} f_{1}^{\prime 2}-2 x f_{1}^{\prime} f_{2}^{\prime}-f_{2}^{\prime 2}\right)\right), \\
& 0=f_{1}^{4}-\beta\left(x f_{1}^{\prime}+f_{2}^{\prime}\right)^{2}+f_{1}^{2}\left(-1+\beta-x^{2} f_{1}^{\prime 2}-2 x f_{1}^{\prime} f_{2}^{\prime}-f_{2}^{\prime 2}\right)
\end{aligned}
$$




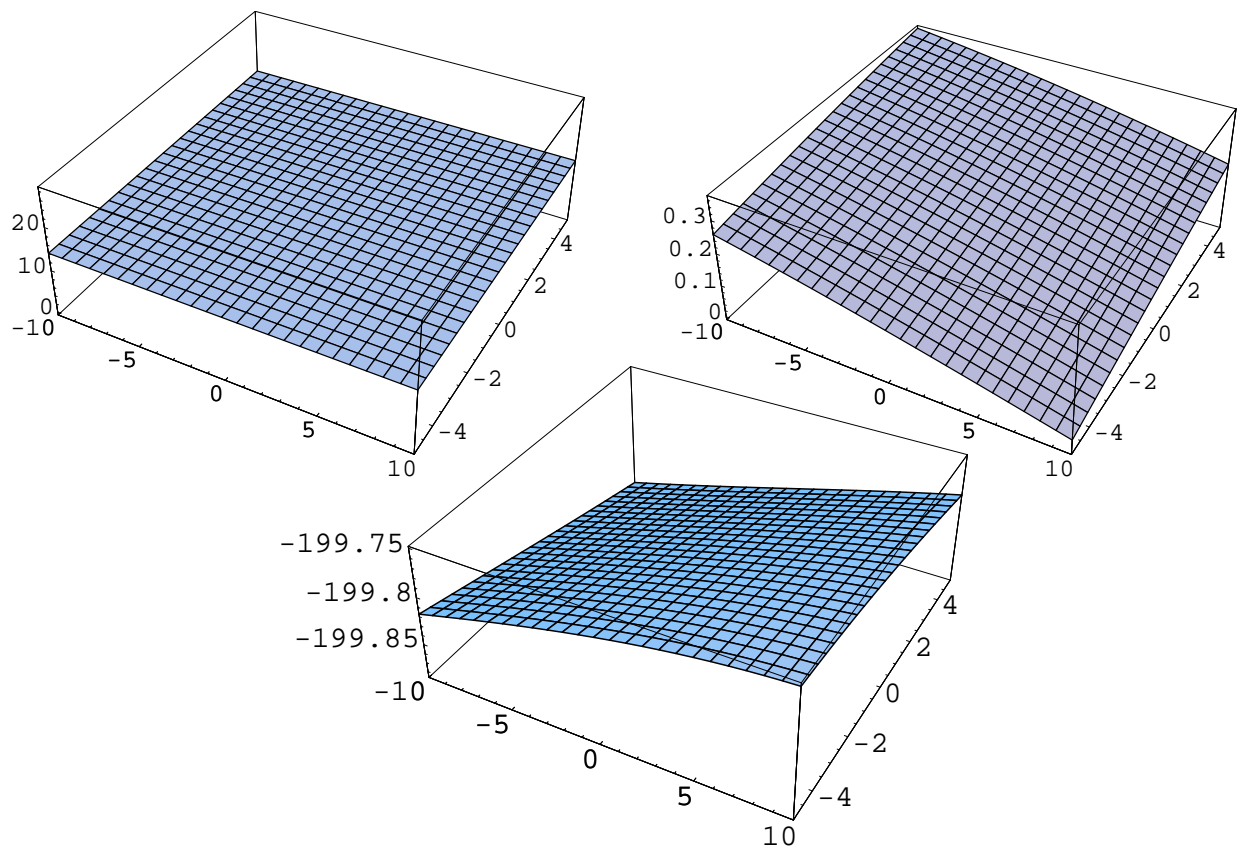

Figure 4. Clockwise from top left: Graphs of $u(x, t), v(x, t)$ and $\eta(x, t)$ for $\beta=-1, c_{1}=0.05 i$ and $c_{2}=-i$.

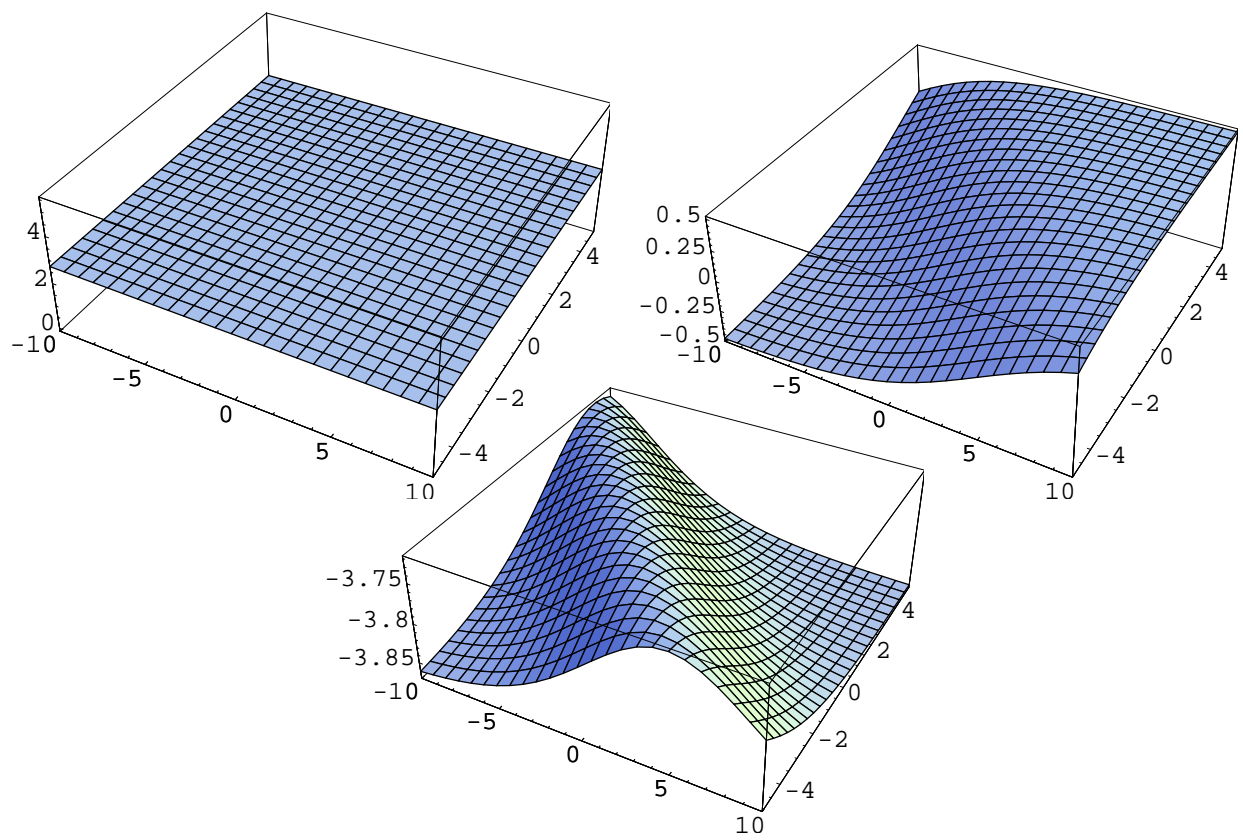

Figure 5. Clockwise from top left: Graphs of $u(x, t), v(x, t)$ and $\eta(x, t)$ for $\beta=-0.5, c_{1}=-0.3$ and $c_{2}=-i$. 
Equating the coefficients of $x^{2}, x^{1}$ and $x^{0}$ in the second of these to zero, we obtain

$$
\begin{aligned}
& 0=-f_{1}^{2}+\beta f_{1}^{2}+f_{1}^{4}-\beta f_{2}^{\prime 2}-f_{1}^{2} f_{2}^{\prime 2}, \\
& 0=-2 \beta f_{1}^{\prime} f_{2}^{\prime}-2 f_{1}^{2} f_{1}^{\prime} f_{2}^{\prime}, \\
& 0=-f_{1}^{\prime 2}\left(\beta+f_{1}^{2}\right) .
\end{aligned}
$$

The latter two are satisfied if $f_{1}(t)=c_{1}$. Substituting this into the first equation above and solving for $f_{2}$, we get

$$
f_{2}(t)=t \sqrt{\frac{-c_{1}^{2}\left(c_{1}^{2}+\beta-1\right)}{-\beta-c_{1}^{2}}}+c_{2} .
$$

Substituting all the above results into Equations (10), (14) and (19) and solving for $v_{1}, u_{1}$ and $\eta_{1}$ we find

$$
\begin{aligned}
& u_{1}(x, t)=-1 / 2, \\
& v_{1}(x, t)=\frac{i \sqrt{\left(-\beta-c_{1}^{2}+1\right)}}{2 c_{1}\left(\beta+c_{1}^{2}\right)}, \\
& \eta_{1}(x, t)=-\left(\beta+c_{1}^{2}\right) \exp (i h(x, t)),
\end{aligned}
$$

where $h(x, t)=c_{1} x+c_{2}+t \sqrt{c_{1}^{2}\left(1-\beta-c_{1}^{2}\right)} / \sqrt{-\beta-c_{1}^{2}}$. Solving (8) or (12) or (13) for $\eta_{2}$, we get

$$
\eta_{2}(x, t)=\frac{\beta\left(1-c_{1}^{2}+c_{1}^{4}\right)-\beta^{2}\left(1-c_{1}^{2}\right)}{4 c_{1}^{2}\left(\beta+c_{1}^{2}\right)},
$$

Substituting all the results into the truncated expansion (7), we obtain the corresponding exact solution of GZEs (3):

$$
\begin{aligned}
v(x, t) & =\frac{i \sqrt{1-\beta-c_{1}^{2}}}{2 c_{1} \sqrt{-\beta-c_{1}^{2}}} \\
u(x, t) & =-\frac{1}{2}+\frac{\exp (i h(x, t))}{1+\exp (i h(x, t))} \\
\eta(x, t) & =\frac{\beta\left(1-c_{1}^{2}+c_{1}^{4}\right)-\beta^{2}\left(1-c_{1}^{2}\right)}{4 c_{1}^{2}\left(\beta+c_{1}^{2}\right)} \\
& +\frac{\left(\beta+c_{1}^{2}\right) \exp (i h(x, t))}{1+\exp (i h(x, t))}\left(\frac{\exp (i h(x, t))}{1+\exp (i h(x, t))}-1\right) .
\end{aligned}
$$

Figures $6,7,8,9$ and 10 illustrate the solution for certain $\beta, c_{1}$ and $c_{2}$. 


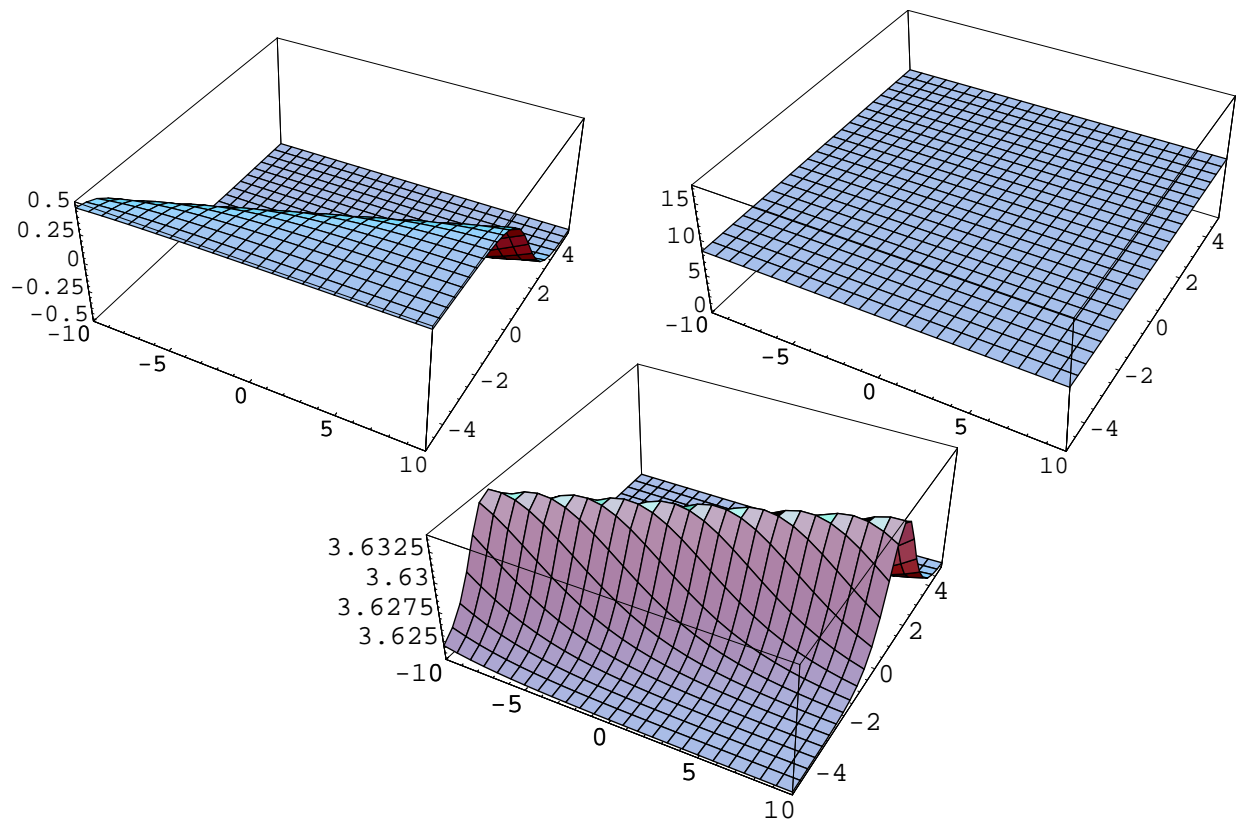

Figure 6. Clockwise from top left: Graphs of $u(x, t), v(x, t)$ and $\eta(x, t)$ for $\beta=0.05, c_{1}=-0.3 i$ and $c_{2}=i$.

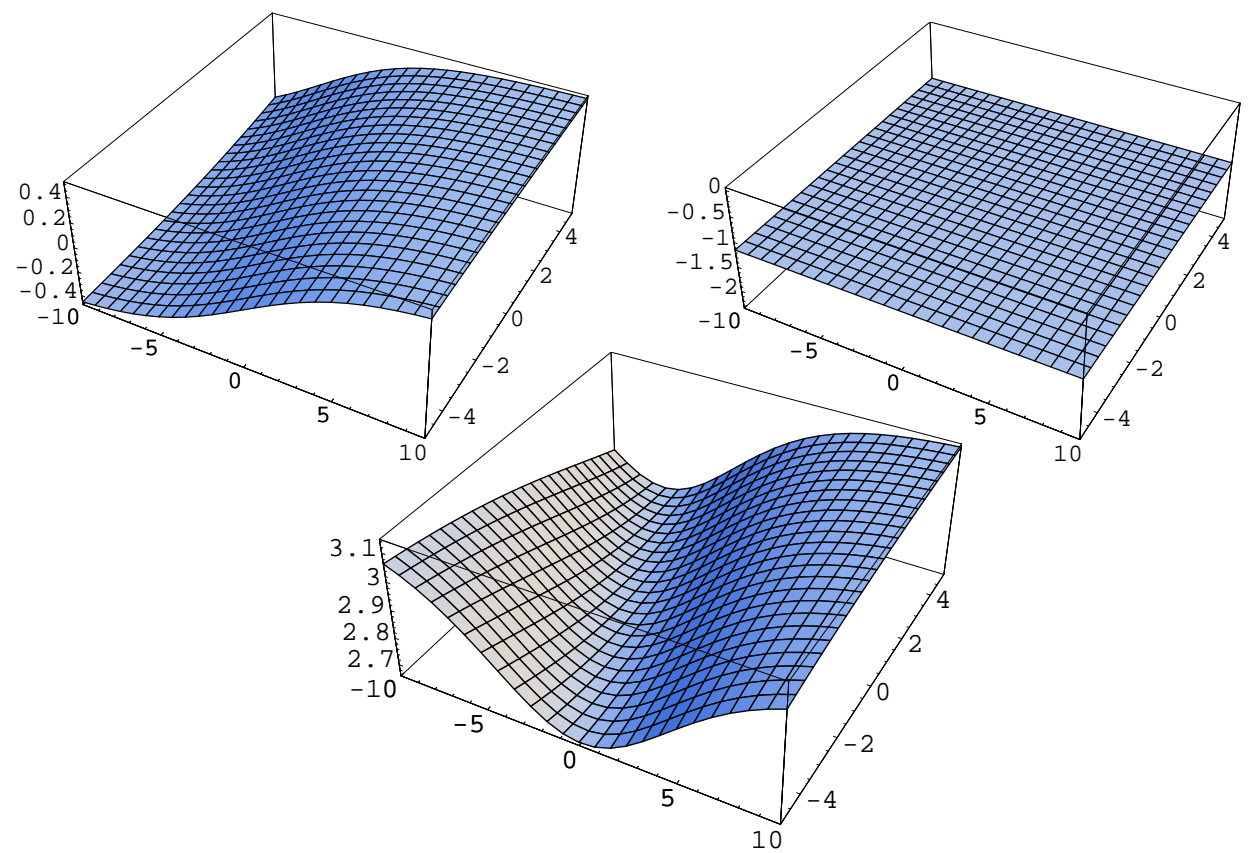

Figure 7. Clockwise from top left: Graphs of $u(x, t), v(x, t)$ and $\eta(x, t)$ for $\beta=2, c_{1}=-0.3 i$ and $c_{2}=-i$. 


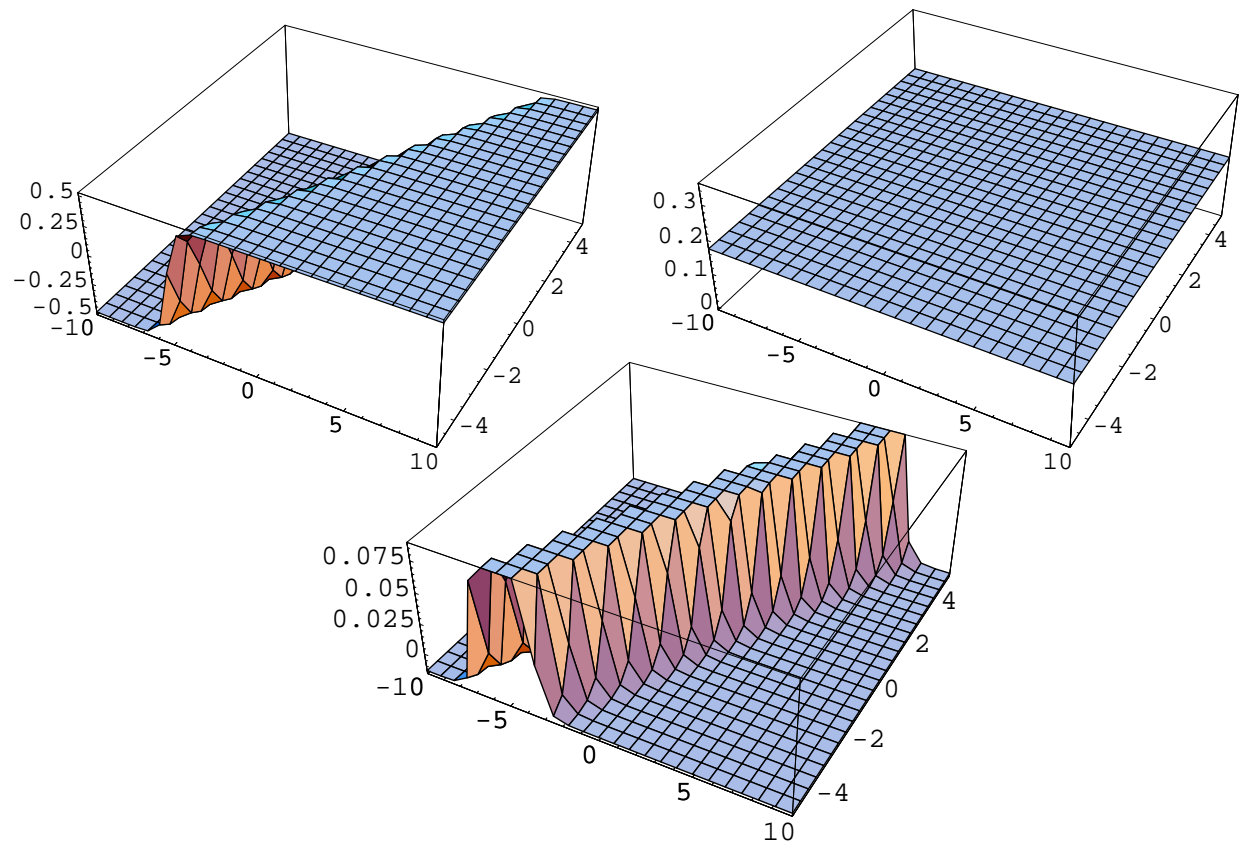

Figure 8. Clockwise from top left: Graphs of $u(x, t), v(x, t)$ and $\eta(x, t)$ for $\beta=-0.05, c_{1}=-3 i$ and $c_{2}=0.4 i$.

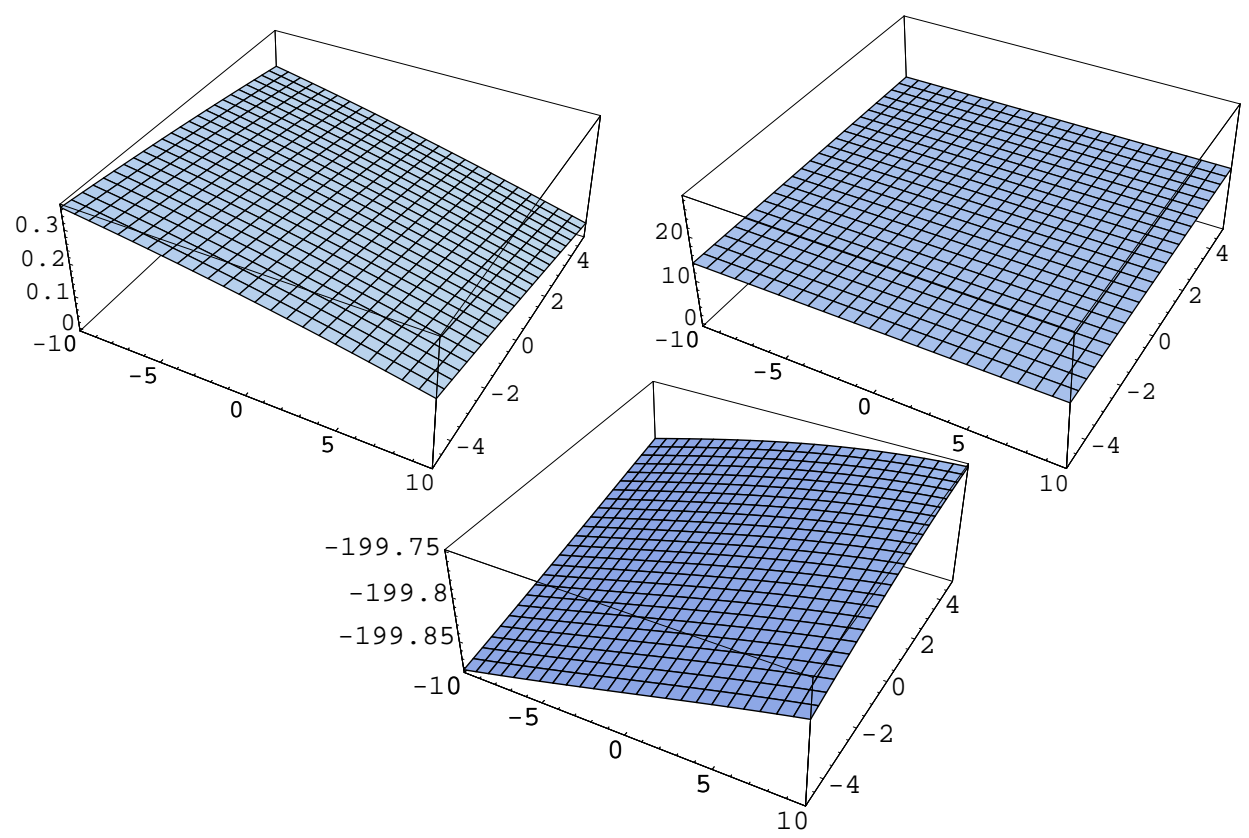

Figure 9. Clockwise from top left: Graphs of $u(x, t), v(x, t)$ and $\eta(x, t)$ for $\beta=-1, c_{1}=0.05$ and $c_{2}=-i$. 


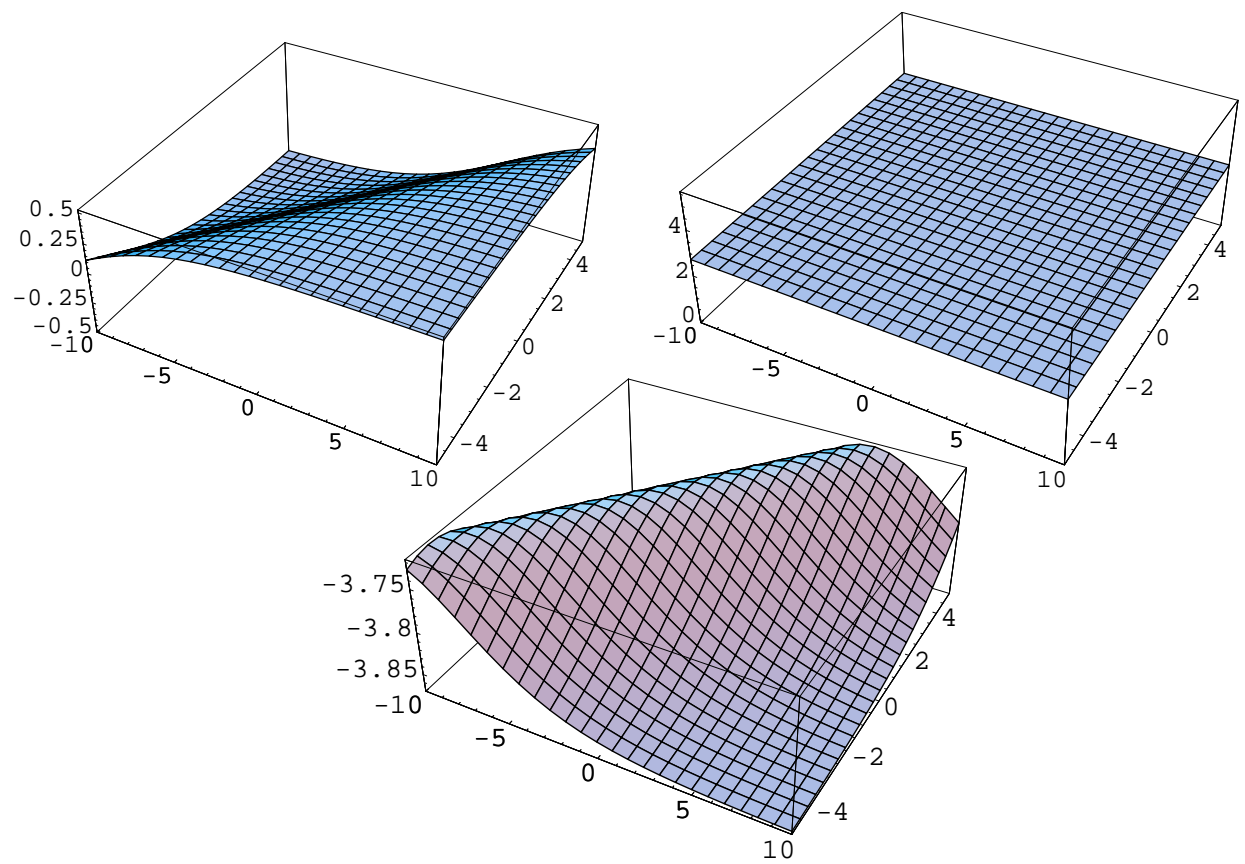

Figure 10. Clockwise from top left: Graphs of $u(x, t), v(x, t)$ and $\eta(x, t)$ for $\beta=-0.5, c_{1}=-0.3$ and $c_{2}=-i$.

\section{References}

[Abbasbandy et al. 2009] S. Abbasbandy, E. Babolian, and M. Ashtiani, "Numerical solution of the generalized Zakharov equation by homotopy analysis method", Commun. Nonlinear Sci. Numer. Simul. 14:12 (2009), 4114-4121. MR 2537572 Zbl 1159.65348

[Bluman and Kumei 1989] G. W. Bluman and S. Kumei, Symmetries and differential equations, Applied Mathematical Sciences 81, Springer, New York, 1989. MR 91b:58284 Zbl 0698.35001

[Choudhury 2006] S. R. Choudhury, "Painlevé analysis of nonlinear evolution equations — an algorithmic method", Chaos Solitons Fractals 27:1 (2006), 139-152. MR 2006b:37132 Zbl 1088.35553

[Hadouaj et al. 1991] H. Hadouaj, B. A. Malomed, and G. A. Maugin, "Dynamics of a soliton in a generalized Zakharov system with dissipation", Phys. Rev. A (3) 44:6 (1991), 3925-3931. MR 92g:35190

[Javidi and Golbabi 2008] M. Javidi and A. Golbabi, "Exact and numerical solitary wave solutions of generalized Zakharov equation by the variational iteration method", Chaos, Solitons and Fractals 36 (2008), 309-313. Zbl 05235317

[Li et al. 2008] Y.-Z. Li, K.-M. Li, and C. Lin, "Exp-function method for solving the generalized Zakharov equations", Appl. Math. Comput. 205:1 (2008), 197-201. MR 2466623 Zbl 1160.35523

[Malomed et al. 1997] B. Malomed, D. Anderson, M. Lisak, M. Quiroga-Teixeiro, and L. Stenflo, "Dynamics of solitary waves in the Zakharov model equations", Phys. Rev. E 55 (1997), 962-968.

[Myrzakulov 1999] R. Myrzakulov, "Singularity structure analysis, integrability solitons and dromions in $(2+1)$-dimensional Zakharov equations", preprint, 1999. arXiv solv-int/9903008 
[Wang and Li 2005] M. Wang and X. Li, "Extended $F$-expansion method and periodic wave solutions for the generalized Zakharov equations", Phys. Lett. A 343 (2005), 48-54. MR 2005m:35266 Zbl 1181.35255

[Wang et al. 2007] Y.-Y. Wang, C.-Q. Dai, L. Wu, and J.-F. Zhang, "Exact and numerical solitary wave solutions of generalized Zakharov equation by the Adomian decomposition method", Chaos Solitons Fractals 32:3 (2007), 1208-1214. MR 2007h:35322 Zbl 1130.35120

[Weiss et al. 1983] J. Weiss, M. Tabor, and G. Carnevale, "The Painlevé property for partial differential equations", J. Math. Phys. 24:3 (1983), 522-526. MR 84c:35101 Zbl 0514.35083

[Zhang 2007] H. Zhang, "New exact travelling wave solutions of the generalized Zakharov equations", Rep. Math. Phys. 60:1 (2007), 97-106. MR 2008i:35233 Zbl 1170.35524

Received June 23, 2009. Revised January 10, 2010.

HASSAN A. ZEDAN

MATHEMATICS DEPARTMENT

KAFR EL-SHEIKH UNIVERSITY

KAFR EL-SHEIKH

EGYPT

Current address:

Mathematics Department

KING ABDUl AZIZ UNIVERSITY

PO BOX 80203

JEDDAH 21589

SAUDI ARABIA

hassanzedan2003@yahoo.com

SALMA M. Al-TUWAIRQI

Mathematics Department

KING ABDULAZIZ UNIVERSITY

JEDDAH 21589

SAUDI ARABIA

saltuwairqi@gmail.com 


\title{
PACIFIC JOURNAL OF MATHEMATICS
}

\author{
http://www.pjmath.org \\ Founded in 1951 by \\ E. F. Beckenbach (1906-1982) and F. Wolf (1904-1989)
}

\section{EDITORS}

V. S. Varadarajan (Managing Editor)

Department of Mathematics

University of California

Los Angeles, CA 90095-1555

pacific@math.ucla.edu

Vyjayanthi Chari

Department of Mathematics

University of California

Riverside, CA 92521-0135

chari@math.ucr.edu

Robert Finn

Department of Mathematics Stanford University

Stanford, CA 94305-2125

finn@math.stanford.edu

Kefeng Liu

Department of Mathematics

University of California

Los Angeles, CA 90095-1555

liu@math.ucla.edu
Darren Long

Department of Mathematics

University of California

Santa Barbara, CA 93106-3080

long@math.ucsb.edu

Jiang-Hua Lu

Department of Mathematics

The University of Hong Kong

Pokfulam Rd., Hong Kong jhlu@maths.hku.hk

Alexander Merkurjev

Department of Mathematics

University of California

Los Angeles, CA 90095-1555

merkurev@math.ucla.edu
Sorin Popa

Department of Mathematics University of California

Los Angeles, CA 90095-1555 popa@math.ucla.edu

Jie Qing

Department of Mathematics

University of California

Santa Cruz, CA 95064

qing@cats.ucsc.edu

Jonathan Rogawski

Department of Mathematics

University of California

Los Angeles, CA 90095-1555

jonr@math.ucla.edu

\section{PRODUCTION}

pacific@math.berkeley.edu

\begin{abstract}
Silvio Levy, Scientific Editor Matthew Cargo, Senior Production Editor
\end{abstract}
ACADEMIA SINICA, TAIPEI

CALIFORNIA INST. OF TECHNOLOGY

INST. DE MATEMÁTICA PURA E APLICADA

KEIO UNIVERSITY

MATH. SCIENCES RESEARCH INSTITUTE

NEW MEXICO STATE UNIV.

OREGON STATE UNIV.

\section{SUPPORTING INSTITUTIONS}

STANFORD UNIVERSITY
UNIV. OF BRITISH COLUMBIA
UNIV. OF CALIFORNIA, BERKELEY
UNIV. OF CALIFORNIA, DAVIS
UNIV. OF CALIFORNIA, LOS ANGELES
UNIV. OF CALIFORNIA, RIVERSIDE
UNIV. OF CALIFORNIA, SAN DIEGO
UNIV. OF CALIF., SANTA BARBARA

UNIV. OF CALIF., SANTA CRUZ

UNIV. OF MONTANA

UNIV. OF OREGON

UNIV. OF SOUTHERN CALIFORNIA

UNIV. OF UTAH

UNIV. OF WASHINGTON

WASHINGTON STATE UNIVERSITY

These supporting institutions contribute to the cost of publication of this Journal, but they are not owners or publishers and have no responsibility for its contents or policies.

See inside back cover or www.pjmath.org for submission instructions.

The subscription price for 2010 is US \$420/year for the electronic version, and \$485/year for print and electronic.

Subscriptions, requests for back issues from the last three years and changes of subscribers address should be sent to Pacific Journal of Mathematics, P.O. Box 4163, Berkeley, CA 94704-0163, U.S.A. Prior back issues are obtainable from Periodicals Service Company, 11 Main Street, Germantown, NY 12526-5635. The Pacific Journal of Mathematics is indexed by Mathematical Reviews, Zentralblatt MATH, PASCAL CNRS Index, Referativnyi Zhurnal, Current Mathematical Publications and the Science Citation Index.

The Pacific Journal of Mathematics (ISSN 0030-8730) at the University of California, c/o Department of Mathematics, 969 Evans Hall, Berkeley, CA 94720-3840, is published monthly except July and August. Periodical rate postage paid at Berkeley, CA 94704, and additional mailing offices. POSTMASTER: send address changes to Pacific Journal of Mathematics, P.O. Box 4163, Berkeley, CA 94704-0163.

PJM peer review and production are managed by EditFLOW ${ }^{\mathrm{TM}}$ from Mathematical Sciences Publishers.

PUBLISHED BY PACIFIC JOURNAL OF MATHEMATICS

at the University of California, Berkeley 94720-3840

A NON-PROFIT CORPORATION

Typeset in LATEX

Copyright $(\mathrm{C} 2010$ by Pacific Journal of Mathematics 


\section{PACIFIC JOURNAL OF MATHEMATICS}

Volume $247 \quad$ No. $2 \quad$ October 2010

A family of representations of braid groups on surfaces

257

ByUnG HeE AN and Ki HyOUNG Ko

Parametrization of holomorphic Segre-preserving maps

283

R. BLAIR ANGLE

Chern classes on differential $K$-theory

ULRICH BUNKE

Laplacian spectrum for the nilpotent Kac-Moody Lie algebras

DMITRY FUCHS and CONSTANCE WILMARTH

Sigma theory and twisted conjugacy classes

DACIBERG GONÇALVES and DESSISLAVA Hristova Kochloukova

Properties of annular capillary surfaces with equal contact angles

JAMES GORDON and DAVID SIEGEL

Approximating annular capillary surfaces with equal contact angles

JAMES GORDON and DAVID SIEGEL

Harmonic quasiconformal self-mappings and Möbius transformations of the unit ball

David Kalaj and Miodrag S. Mateljević

Klein bottle and toroidal Dehn fillings at distance 5

SANGYOP LEE

Representations of the two-fold central extension of $\mathrm{SL}_{2}\left(\mathbb{Q}_{2}\right)$

HUNG YEAN LOKE and GORDAN SAVIN

Large quantum corrections in mirror symmetry for a 2-dimensional

Lagrangian submanifold with an elliptic umbilic

Giovanni MARELLI

Crossed pointed categories and their equivariantizations

DEEPAK NAIDU

Painlevé analysis of generalized Zakharov equations

Hassan A. ZeDan and Salma M. Al-Tuwairqi 